\title{
Plasma Neutrophil Gelatinase-Associated Lipocalin as a Predictor of Cardiovascular Events in Patients with Chronic Kidney Disease
}

\author{
Midori Hasegawa, ${ }^{1}$ Junichi Ishii, ${ }^{2}$ Fumihiko Kitagawa, ${ }^{3}$ Hiroshi Takahashi, ${ }^{4}$ \\ Kazuhiro Sugiyama, ${ }^{1}$ Masashi Tada, ${ }^{1}$ Kyoko Kanayama, ${ }^{1}$ Kazuo Takahashi, ${ }^{1}$ \\ Hiroki Hayashi, ${ }^{1}$ Shigehisa Koide, ${ }^{1}$ Shigeru Nakai, ${ }^{1}$ Yukio Ozaki, ${ }^{5}$ and Yukio Yuzawa ${ }^{1}$ \\ ${ }^{1}$ Department of Nephrology, Fujita Health University School of Medicine, 1-98 Dengakugakubo, Kutsukakecho, Toyoakeshi, \\ Aichi 470-1192, Japan \\ ${ }^{2}$ Laboratory of Clinical Medicine, Department of Joint Research, Fujita Health University School of Medicine, \\ 1-98 Dengakugakubo, Kutsukakecho, Toyoakeshi, Aichi 470-1192, Japan \\ ${ }^{3}$ Laboratory of Clinical Medicine, Fujita Health University, 1-98 Dengakugakubo, Kutsukakecho, Toyoakeshi, Aichi 470-1192, Japan \\ ${ }^{4}$ Division of Medical Statistics, Fujita Health University Hospital, 1-98 Dengakugakubo, Kutsukakecho, Toyoakeshi, \\ Aichi 470-1192, Japan \\ ${ }^{5}$ Department of Cardiology, Fujita Health University School of Medicine, 1-98 Dengakugakubo, Kutsukakecho, Toyoakeshi, \\ Aichi 470-1192, Japan
}

Correspondence should be addressed to Midori Hasegawa; mhase@fujita-hu.ac.jp

Received 29 August 2015; Revised 11 February 2016; Accepted 24 February 2016

Academic Editor: Maciej Banach

Copyright (c) 2016 Midori Hasegawa et al. This is an open access article distributed under the Creative Commons Attribution License, which permits unrestricted use, distribution, and reproduction in any medium, provided the original work is properly cited.

Background. Our aim was to assess plasma neutrophil gelatinase-associated lipocalin (NGAL) as a predictor of cardiovascular (CV) events in patients with chronic kidney disease (CKD) and no history of CV events. Methods. This was a prospective observational cohort study of 252 patients with predialysis CKD. CV events were defined as CV death, acute coronary syndrome, and hospitalization for worsening heart failure, stroke, and aortic dissection. Results. During a median follow-up period of 63 months, $36 \mathrm{CV}$ events occurred. On Cox stepwise multivariate analysis, plasma NGAL and B-type natriuretic peptide (BNP) were significant predictors of CV events. Kaplan-Meier incidence rates of CV event-free survival at 5 years were $96.6 \%, 92.9 \%, 85.9 \%$, and $61.3 \%$, respectively, among quartiles of plasma NGAL $(P<0.0001)$. The $\mathrm{C}$-index for the receiver-operating characteristic curves for CV events was greater when plasma NGAL was added to an established risk model (0.801, 95\% CI 0.717-0.885), compared to the model without plasma NGAL $(0.746,95 \%$ CI $0.653-0.840, P=0.021)$. Conclusion. Elevated plasma NGAL could predict future $\mathrm{CV}$ events in CKD patients with no history of CV events and add incremental value to the established risk model.

\section{Introduction}

Individuals with chronic kidney disease $(\mathrm{CKD})$ are at extremely high cardiovascular (CV) risk [1-4]. CKD contributes to decreased cardiac function, ventricular hypertrophy, and diastolic dysfunction [5]. Biomarkers associated with adverse $\mathrm{CV}$ events are useful to detect high risk patients [6-8]. Human neutrophil gelatinase-associated lipocalin (NGAL) was initially identified as a protein isolated from the secondary granules of human neutrophils [9]. In 2003, Mishra et al. reported that NGAL could be detected in the first urine output after ischemia, in both mouse and rat models of acute renal failure [10]. In 2005, Mori et al. clarified that NGAL accumulates in the human kidney cortical tubules and in the blood and urine after nephrotoxic and ischemic injury [11], and Mishra et al. reported that the NGAL concentration in urine and serum represents a sensitive, specific, and highly predictive early biomarker for 
acute renal injury after cardiac surgery [12]. In the ensuing years, many studies have reported the utility of NGAL as a biomarker of acute kidney injury (AKI) [13].

Recent studies have reported that NGAL may be a biomarker for heart failure as well as renal failure [14, 15]. However, research has not addressed the clinical significance of plasma NGAL as a biomarker of CV risk in patients with CKD who are not on dialysis. The purpose of this study is to evaluate plasma NGAL as a predictor of CV events in patients with CKD.

\section{Patients and Methods}

2.1. Study Participants. This prospective cohort study was approved by the Ethics Committee of the Fujita Health University School of Medicine. Patients were enrolled between February 2009 and September 2010. Subjects were eligible for inclusion if they met the following criteria: (1) diagnosis of CKD, defined as having an estimated glomerular filtration rate (eGFR) lower than $60 \mathrm{~mL} / \mathrm{min} / 1.73 \mathrm{~m}^{2}$; (2) not receiving dialysis; and (3) no history of $\mathrm{CV}$ events. $\mathrm{CV}$ events regarding past medical history and outcome assessment were defined as acute coronary syndrome, hospitalization for worsening heart failure, stroke, and dissection of aorta, and CV death. Patients were excluded if they presented with fever or signs of infectious diseases, for less than one year. The eGFR was calculated using the Modification of Diet in Renal Disease Study formula for the Japanese population [16].

2.2. Measurement of Biomarkers. Blood samples collected for measurement of plasma NGAL were centrifuged at $1000 \times \mathrm{g}$ at $4^{\circ} \mathrm{C}$ for 15 minutes and stored at $-80^{\circ} \mathrm{C}$ until assayed. Urine samples were also stored at $-80^{\circ} \mathrm{C}$ until assayed. NGAL was measured using enzyme-linked immunosorbent assays (BioPorto Diagnostics, Gentofte, Denmark).

2.3. Outcomes Assessment. All patients were observed clinically from enrollment until conclusion of follow-up in December 2014. CV events were assessed by physicians who were blinded to patients' plasma and urinary NGAL levels. Patients were identified as having diabetes if their medical records contained documentation of previous history of diabetes, diagnosis of diabetes on admission, or use of an oral antihyperglycemic agent or insulin at the time of hospital admission.

2.4. Statistical Analyses. Statistical analyses were conducted using the Statistical Package for the Social Sciences, Version 19.0 for Windows (SPSS, Chicago, IL, USA). Data are presented as medians and interquartile ranges (IQR). The relationship between the plasma NGAL and other baseline valuables was studied by linear regression analysis. Multivariate regression model including variables with $P<0.05$ in the individual model was used to determine which covariates were independently associated with log plasma NGAL levels. Differences in event-free survival by quartile of plasma NGAL were examined using the Kaplan-Meier method and compared using a log-rank test. Hazard ratios (HR) and 95\%
TABLE 1: Clinical characteristicof 252 CKD patients.

\begin{tabular}{|c|c|}
\hline & All patients $(n=252)$ \\
\hline Male (\%) & 59.1 \\
\hline Age (years) & $67(60-75)$ \\
\hline Diabetes (\%) & 35.7 \\
\hline Smoking (\%) & 37.9 \\
\hline Systolic BP (mmHg) & $130(122-142)$ \\
\hline BMI $\left(\mathrm{kg} / \mathrm{m}^{2}\right)$ & $22.3(20.2-24.7)$ \\
\hline \multicolumn{2}{|l|}{ CKD grade (\%) } \\
\hline G3a & 25.4 \\
\hline G3b & 14.7 \\
\hline G4 & 35.7 \\
\hline G5 & 24.2 \\
\hline Plasma NGAL (ng/mL) & $105(60-194)$ \\
\hline Urinary NGAL ( $\mu \mathrm{g} / \mathrm{gCr})$ & $65(15-235)$ \\
\hline BNP (pg/mL) & $30(17-71)$ \\
\hline $\mathrm{eGFR}\left(\mathrm{mL} / \mathrm{min} / 1.73 \mathrm{~m}^{2}\right)$ & $25.1(15.2-38.5)$ \\
\hline Hemoglobin (g/dL) & $11.3(9.8-12.7)$ \\
\hline hsCRP (mg/L) & $0.6(0.2-1.5)$ \\
\hline UACR (mg/gCr) & $338(102-1321)$ \\
\hline $\mathrm{TG}(\mathrm{mg} / \mathrm{dL})$ & $137(103-193)$ \\
\hline LDL-C (mg/dL) & $109(87-129)$ \\
\hline HDL-C (mg/dL) & $54(44-68)$ \\
\hline \multicolumn{2}{|l|}{$\mathrm{ECG}^{\mathrm{a}}$} \\
\hline LVH (\%) & 6.1 \\
\hline ST-T change (\%) & 9.8 \\
\hline $\mathrm{EF}(\%)$ by $\mathrm{UCG}^{\mathrm{b}}$ & $60.0(57.0-64.0)$ \\
\hline ARB or ACEi (\%) & 71.0 \\
\hline Statins (\%) & 29.8 \\
\hline
\end{tabular}

${ }^{\mathrm{a}} n=194 ;{ }^{\mathrm{b}} n=151$.

$\mathrm{ACEi}$, angiotensin-converting enzyme inhibitor; $\mathrm{ARB}$, angiotensin receptor blocker; BP, blood pressure; BMI, body mass index; BNP, B-type natriuretic peptide; ECG, electrocardiogram; CKD, chronic kidney disease; EF, ejection fraction; eGFR, estimated glomerular filtration rate; HDL-C, high-density lipoprotein-cholesterol; hsCRP, high-sensitivity C-reactive protein; LDLC, low-density lipoprotein-cholesterol; LVH, left ventricular hypertrophy; NGAL, neutrophil gelatinase-associated lipocalin; TG, triglyceride; UACR, urinary albumin creatinine ratio; UCG, ultracardiography.

confidence intervals (CI) were calculated for each factor with Cox proportional hazards analysis. To identify independent predictors of $\mathrm{CV}$ events, all baseline variables with $P<0.05$ in the univariate analysis were entered into a multivariate model. In addition, to assess if $\mathrm{CV}$ events could be predicted more accurately after the addition of plasma NGAL to the model, the $\mathrm{C}$-index for the receiver-operating characteristic (ROC) curves was calculated using a logistic multivariate model. Differences were considered statistically significant at $P<0.05$.

\section{Results}

3.1. Study Population. The study enrolled 252 patients. Characteristics of the study population are summarized in Table 1. The median age of patients was $67(60-75)$ years, and $59.1 \%$ were male. The distribution of patients among the CKD 
TABLE 2: Individual and multivariable covariates of log plasma NGAL levels.

\begin{tabular}{lcccc}
\hline \multirow{2}{*}{ Variable } & \multicolumn{2}{c}{ Individual } & \multicolumn{2}{c}{ Multivariate $^{*}$} \\
& $r$ & $P$ & $\beta$ & $P$ \\
\hline Age & 0.063 & 0.32 & & \\
Systolic BP & 0.14 & 0.024 & & \\
BMI & 0.043 & 0.52 & & \\
log urinary NGAL & 0.56 & $<0.0001$ & & \\
$\operatorname{logBNP}$ & 0.34 & $<0.0001$ & 0.12 & 0.010 \\
$\operatorname{logeGFR}$ & -0.73 & $<0.0001$ & -0.59 & $<0.0001$ \\
$\operatorname{logHb}$ & -0.50 & $<0.0001$ & & \\
$\operatorname{loghsCRP}$ & 0.064 & 0.31 & & \\
$\log \mathrm{UACR}$ & 0.45 & $<0.0001$ & 0.17 & 0.001 \\
$\log$ TG & -0.090 & 0.16 & & \\
$\operatorname{logLDL}-\mathrm{C}$ & -0.13 & 0.049 & & \\
$\operatorname{logHDL}-\mathrm{C}$ & -0.23 & $<0.0001$ & -0.20 & $<0.0001$ \\
EF (\%) by UCG & -0.14 & 0.082 & & \\
\hline
\end{tabular}

$\mathrm{b}_{n=151}$

${ }^{*} R^{2}=0.58 ; \beta=$ standardized regression coefficient.

Multivariate model includes all variables with $P<0.05$ by individual model. $\mathrm{BP}$, blood pressure; BMI, body mass index; BNP, B-type natriuretic peptide; $\mathrm{EF}$, ejection fraction; eGFR, estimated glomerular filtration rate; HDL$\mathrm{C}$, high-density lipoprotein-cholesterol; hsCRP, high-sensitivity C-reactive protein; LDL-C, low-density lipoprotein-cholesterol; NGAL, neutrophil gelatinase-associated lipocalin; TG, triglyceride; UACR, urinary albumin creatinine ratio; UCG, ultracardiography.

grades was $25.4 \%$ of G3a, $14.7 \%$ of $\mathrm{G} 3 \mathrm{~b}, 35.7 \%$ of $\mathrm{G} 4$, and $24.2 \%$ of G5.

3.2. Relationships between Urinary and Plasma Neutrophil Gelatinase-Associated Lipocalin. The scatter plot of log (plasma NGAL) and log (urinary NGAL) is shown in Figure 1 . The correlation coefficient was $0.56, P<0.0001$.

3.3. Correlates of Plasma Neutrophil Gelatinase-Associated Lipocalin Levels. Correlation between clinical and laboratory variables and plasma NGAL levels are shown in Table 2. The strongest individual correlations were observed with eGFR. Moderate associations were found with hemoglobin $(\mathrm{Hb})$, urinary NGAL, and the urinary albumin creatinine ratio (UACR). Weak associations were found with B-type natriuretic peptide (BNP) and high-density lipoprotein- (HDL-) cholesterol. Four variables were independently associated with NGAL levels in the multivariate analysis: eGFR, HDLcholesterol, UACR, and BNP.

3.4. Prognostic Value of Plasma Neutrophil GelatinaseAssociated Lipocalin for Cardiovascular Events. During a median follow-up period of 63 months, $36 \mathrm{CV}$ events $(14.3 \%)$ occurred. Twenty-two consisted of admission due to heart failure, 5 were acute myocardial infarction, 5 were cerebral infarction, 3 were aortic dissection, and 1 was unstable angina pectoris. Table 3 shows Cox analysis for CV events. On univariate analysis, age, plasma NGAL, BNP, eGFR, hemoglobin, high-sensitivity C-reactive protein (hsCRP), and UACR were significant predictors of $\mathrm{CV}$ events. When we performed

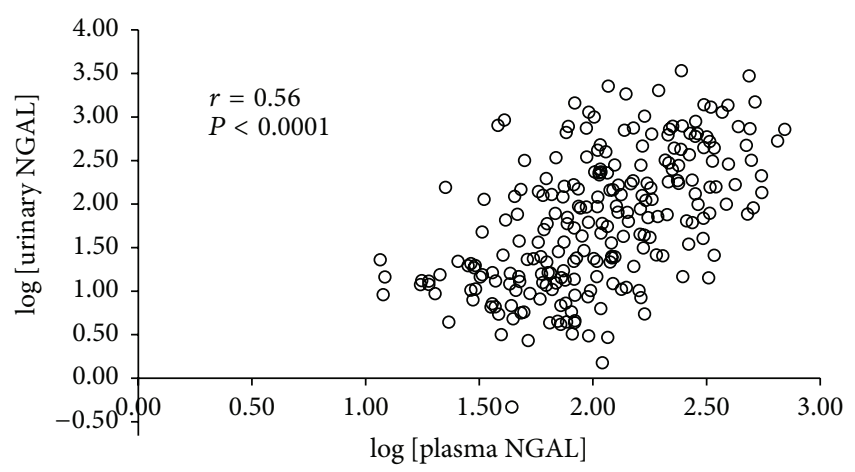

FIgURE 1: Plot of log plasma neutrophil gelatinase-associated lipocalin (NGAL) and log urinary NGAL. There was a significant correlation between plasma and urinary NGAL $(r=0.56, P<$ $0.0001)$.

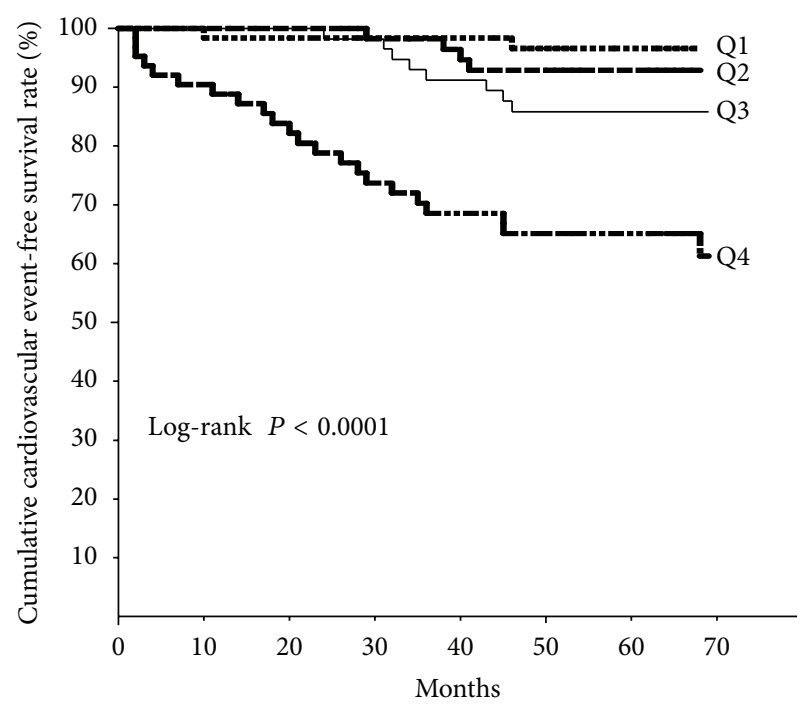

FIgURE 2: Kaplan-Meier curves for cardiovascular event-free survival by quartile (Q1-Q4) of plasma neutrophil gelatinase-associated lipocalin level. A clear difference among quartiles is evident. Quartile 1 (Q1): $\leq 60 \mathrm{ng} / \mathrm{mL}$, quartile 2 (Q2): $61-104 \mathrm{ng} / \mathrm{mL}$, quartile 3 (Q3): $105-193 \mathrm{ng} / \mathrm{mL}$, and quartile 4 (Q4): $\geq 194 \mathrm{ng} / \mathrm{mL}$.

Cox stepwise multivariate analysis including all variables with $P<0.05$ on univariate analysis, plasma NGAL and BNP were significant predictors of CV events. Five-year CV eventfree survival rates in quartile $1(\mathrm{Q} 1 ; \leq 60 \mathrm{ng} / \mathrm{mL})$, quartile 2 (Q2; 61-104 ng/mL), quartile 3 (Q3; 105-193 ng/mL), and quartile $4(\mathrm{Q} 4 ; \geq 194 \mathrm{ng} / \mathrm{mL})$, calculated by the Kaplan-Meier method, were $96.6 \%, 92.9 \%, 85.9 \%$, and $61.3 \%$, respectively $(P<0.0001$, Figure 2$)$.

3.5. Receiver-Operating Characteristic Curves for Cardiovascular Events. Figure 3 shows the ROC curves for CV events. The addition of plasma NGAL to the predictive model based on BNP, hsCRP, UACR, Hb, eGFR, and age had an effect on model discrimination as measured by the C-index $(0.746$ (95\% CI $0.653-0.840)$ to 0.801 (95\% CI $0.717-0.885), P=$ $0.021)$. 
TABLE 3: Predictors of cardiovascular events.

\begin{tabular}{|c|c|c|c|c|}
\hline \multirow{2}{*}{ Variable } & \multicolumn{2}{|c|}{ Nonadjusted } & \multicolumn{2}{|c|}{ Adjusted } \\
\hline & $\operatorname{HR}(95 \% \mathrm{CI})$ & $P$ value & $\operatorname{HR}(95 \% \mathrm{CI})$ & $P$ value \\
\hline Male (yes) & $0.99(0.51-1.95)$ & 1.00 & & \\
\hline Age (yes) & $1.04(1.01-1.07)$ & 0.01 & $1.02(0.99-1.05)$ & 0.24 \\
\hline Diabetes (yes) & $1.87(0.97-3.60)$ & 0.06 & & \\
\hline Smoking (yes) & $0.84(0.42-1.65)$ & 0.61 & & \\
\hline Systolic BP (mmHg) & $1.02(0.99-1.04)$ & 0.12 & & \\
\hline BMI $\left(\mathrm{kg} / \mathrm{m}^{2}\right)$ & $0.95(0.86-1.05)$ & 0.35 & & \\
\hline Plasma NGAL (ng/mL) & $1.005(1.003-1.007)$ & $<0.0001$ & $1.004(1.002-1.006)$ & $<0.0001$ \\
\hline Urinary NGAL $(\mu \mathrm{g} / \mathrm{gCr})$ & $1.00(0.999-1.001)$ & 0.71 & & \\
\hline $\mathrm{BNP}(\mathrm{pg} / \mathrm{mL})$ & $1.005(1.003-1.006)$ & $<0.0001$ & $1.005(1.003-1.007)$ & $<0.0001$ \\
\hline $\mathrm{eGFR}\left(\mathrm{mL} / \mathrm{min} / 1.73 \mathrm{~m}^{2}\right)$ & $0.96(0.93-0.99)$ & 0.002 & $0.99(0.96-1.04)$ & 0.87 \\
\hline Hemoglobin (g/dL) & $0.72(0.60-0.85)$ & $<0.0001$ & $0.94(0.78-1.14)$ & 0.54 \\
\hline hsCRP (mg/L) & $1.78(1.32-2.35)$ & $<0.0001$ & $1.33(0.98-1.82)$ & 0.07 \\
\hline UACR (g/gCr) & $1.26(1.09-1.44)$ & 0.001 & $1.16(0.97-1.39)$ & 0.09 \\
\hline Triglyceride (mg/dL) & $0.99(0.99-1.00)$ & 0.36 & & \\
\hline LDL-C (mg/dL) & $1.00(0.99-1.01)$ & 0.40 & & \\
\hline HDL-C (mg/dL) & $0.99(0.97-1.00)$ & 0.21 & & \\
\hline LVH of ECG ${ }^{\mathrm{a}}$ & $1.75(0.53-5.75)$ & 0.36 & & \\
\hline ST-T change of ECG ${ }^{\mathrm{a}}$ & $1.14(0.35-3.76)$ & 0.83 & & \\
\hline $\mathrm{EF}$ of $\mathrm{UCG}^{\mathrm{b}}$ & $0.94(0.88-1.00)$ & 0.056 & & \\
\hline ARB or ACEi (yes) & $0.66(0.33-1.30)$ & 0.23 & & \\
\hline Statins (yes) & $0.96(0.47-1.95)$ & 0.91 & & \\
\hline
\end{tabular}

${ }^{\mathrm{a}} n=194 ;{ }^{\mathrm{b}} n=151$.

Multivariate model includes all variables with $P<0.05$ by univariate analysis.

$\mathrm{ACEi}$, angiotensin-converting enzyme inhibitor; $\mathrm{ARB}$, angiotensin receptor blocker; $\mathrm{BMI}$, body mass index; $\mathrm{BNP}$, B-type natriuretic peptide; $\mathrm{BP}$, blood pressure; ECG, electrocardiogram; EF, ejection fraction; eGFR, estimated glomerular filtration rate; HDL-C, high-density lipoprotein-cholesterol; HR, hazard ratio; hsCRP, high-sensitivity C-reactive protein; LDL-C, low-density lipoprotein-cholesterol; LVH, left ventricular hypertrophy; NGAL, neutrophil gelatinaseassociated lipocalin; UACR, urinary albumin creatinine ratio; UCG, ultracardiography.

\section{Discussion}

Our primary finding was that plasma NGAL is an independent predictor of CV events. Plasma NGAL therefore provides incremental value to an established risk model that includes $\mathrm{BNP}$, in patients with $\mathrm{CKD}$ and no previous history of $\mathrm{CV}$ events. Neutrophils are the major source of circulating NGAL in both normal and infected states, whereas, during AKI, blood and urinary NGAL derive mainly from the kidney [17]. In an AKI model, NGAL is expressed in the distal nephron. In a study of diabetic mice induced by streptozotocin, which is a model of slowly progressive CKD, urinary NGAL appeared to derive primarily from impaired reabsorption in the proximal tubules [18]. Recent studies indicate that chronic renal damage could influence the physiological balance of NGAL in a manner similar to that observed for acute injury conditions. Chronically damaged tubular cells may produce a large quantity of NGAL because of active chronic stress-induced production by the injured tubular cells [19]. Plasma NGAL measurement has been reported to be influenced by other coexisting factors, including inflammatory conditions [20], anemia, and hypoxia [21]. NGAL expression also occurs in a failing myocardium [22] and is present in atherosclerotic plaque and human abdominal aortic aneurysms [23, 24]. Clinically, divergent results have been observed with regard to ventricular structure and function. Serum NGAL was weakly correlated with left ventricular (LV) ejection fraction in one small study [25], while other studies found no association between plasma or urine NGAL and echocardiographic indices of LV cardiac structure or left or right ventricular systolic function $[22,26]$. In another study, plasma NGAL levels were modestly associated with indices of diastolic dysfunction, but not after adjustment for renal function [22]. In our cohort, plasma NGAL had no significant correlation with LV ejection fraction. Plasma NGAL was independently correlated with eGFR, HDL-cholesterol, UACR, and BNP. Thus plasma NGAL may reflect a variety of factors associated with $\mathrm{CV}$ events.

In terms of the CV-related prognostic value of NGAL, several conflicting results have been reported in different cohorts [24-27]. Lindberg et al. found that high plasma NGAL independently predicts major adverse cardiac events in ST-segment elevation myocardial infarction patients treated with primary percutaneous coronary intervention [27]. Falke et al. reported that plasma NGAL was a significant independent predictor of long-term CV mortality in patients with acute cerebral ischemia [28]. Daniels et al. reported that plasma NGAL was a significant predictor of mortality and $\mathrm{CV}$ events in community-dwelling older adults, independent 


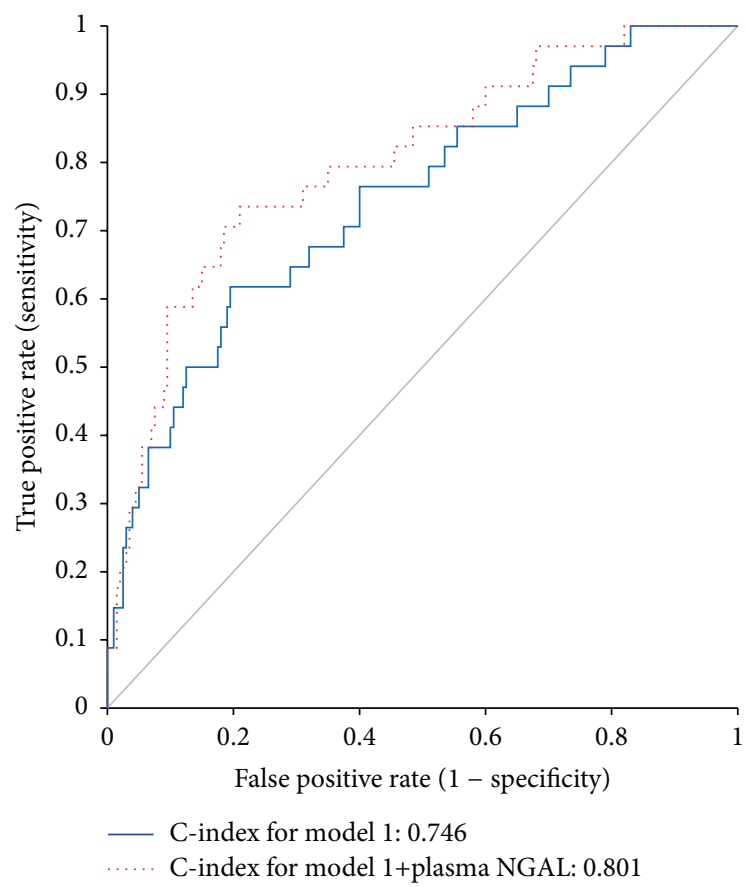

FIGURE 3: Receiver-operating characteristic curve for cardiovascular events in model 1 and model 2. Model 1: BNP + hsCRP + UACR $+\mathrm{Hb}+$ eGFR + age. Model 2: Model $1+$ plasma NGAL. BNP, Btype natriuretic peptide; eGFR, estimated glomerular filtration rate; hsCRP, high-sensitivity C-reactive protein; Hb, hemoglobin; NGAL, neutrophil gelatinase-associated lipocalin; UACR, urinary albumin creatinine ratio.

of traditional risk factors and kidney function, and added incremental value to $\mathrm{N}$-terminal pro-B-type natriuretic peptide (NT-proBNP) and CRP [29]. On the contrary, Nymo et al. reported that NGAL added no significant information to NT-proBNP and GFR in a multivariate model for primary and secondary end-points in chronic heart failure [30]. In our study, we used BNP, rather than NT-proBNP, to detect natriuretic peptide levels. BNP is a neurohormone secreted mainly in the cardiac ventricles in response to volume expansion and pressure overload. Plasma BNP levels have a strong correlation with LV filling pressure and increase in proportion to the severity of LV systolic dysfunction and diastolic dysfunction [31, 32]. Unlike NT-proBNP, plasma BNP levels are relatively independent of GFR. In addition, levels of BNP have been reported to have powerful predictive potential for heart failure in nondialysis CKD patients [33].

Recently, we reported that urinary NGAL was a predictor of $\mathrm{CV}$ events in CKD patients, during a median followup period of 33 months [34]. That study cohort contained patients who had a history of $\mathrm{CV}$ events. In the present study, patients with a history of $\mathrm{CV}$ events were excluded, and the median follow-up period was extended to 63 months. There was a significant correlation between plasma NGAL and urinary NGAL $(r=0.57, P<0.0001)$. However, there were also discrepancies in these two parameters for some patients. Whereas urinary NGAL seems to be closely linked to kidney function and kidney damage, circulating
NGAL levels may also mirror other systemic conditions that we described above. This might explain why urinary NGAL did not remain as a significant predictor of $\mathrm{CV}$ events when adjusting for confounders, including eGFR.

\section{Limitations}

This study has several limitations. First, we used a singlecenter design. Larger multicenter studies might confirm our findings. Second, we could not evaluate any association between NGAL and cardiac death or all-cause mortality because of the small number of deaths. Third, we could not perform separate analysis of each CV end-point, because when separated, each event was too infrequent to be included in multivariate analysis.

\section{Conclusions}

In conclusion, plasma NGAL is an important independent predictor of $\mathrm{CV}$ events in patients with $\mathrm{CKD}$ and no previous CV events. Measuring plasma NGAL could contribute clinically significant information to the evaluation of patients at high risk of CV events.

\section{Competing Interests}

The authors declare that they have no competing interests.

\section{Acknowledgments}

This work was supported by Japan Society for the Promotion of Science KAKENHI, Grant no. 24590712. This work was also supported by a grant-in-aid for Diabetic Nephropathy and Nephrosclerosis Research and for Progressive Renal Diseases Research, Research on Intractable Disease, from the Ministry of Health, Labor and Welfare of Japan, and by the Aichi Kidney Foundation.

\section{References}

[1] R. N. Foley, P. S. Parfrey, and M. J. Sarnak, "Clinical epidemiology of cardiovascular disease in chronic renal disease," American Journal of Kidney Diseases, vol. 32, no. 5, supplement 3, pp. S112-S119, 1998.

[2] D. S. Keith, G. A. Nichols, C. M. Gullion, J. B. Brown, and D. H. Smith, "Longitudinal follow-up and outcomes among a population with chronic kidney disease in a large managed care organization," Archives of Internal Medicine, vol. 164, no. 6, pp. 659-663, 2004.

[3] A. S. Go, G. M. Chertow, D. Fan, C. E. McCulloch, and C.-Y. Hsu, "Chronic kidney disease and the risks of death, cardiovascular events, and hospitalization," The New England Journal of Medicine, vol. 351, no. 13, pp. 1296-1370, 2004.

[4] T. Ninomiya, Y. Kiyohara, M. Kubo et al., "Chronic kidney disease and cardiovascular disease in a general Japanese population: the Hisayama Study," Kidney International, vol. 68, no. 1, pp. 228-236, 2005. 
[5] C. Ronco, M. Cicoira, and P. A. McCullough, "Cardiorenal syndrome type 1: pathophysiological crosstalk leading to combined heart and kidney dysfunction in the setting of acutely decompensated heart failure," Journal of the American College of Cardiology, vol. 60, no. 12, pp. 1031-1042, 2012.

[6] R. Tagore, L. H. Ling, H. Yang, H.-Y. Daw, Y.-H. Chan, and S. K. Sethi, "Natriuretic peptides in chronic kidney disease," Clinical Journal of the American Society of Nephrology, vol. 3, no. 6, pp. 1644-1651, 2008.

[7] M. J. Sarnak, R. Katz, A. Newman et al., "Association of urinary injury biomarkers with mortality and cardiovascular events," Journal of the American Society of Nephrology, vol. 25, no. 7, pp. 1545-1553, 2014.

[8] M. Kuoppamäki, M. Salminen, T. Vahlberg, K. Irjala, S.L. Kivelä, and I. Räihä, "High sensitive C-reactive protein (hsCRP), cardiovascular events and mortality in the aged: a prospective 9-year follow-up study," Archives of Gerontology and Geriatrics, vol. 60, no. 1, pp. 112-117, 2015.

[9] S. Y. Xu, M. Carlson, A. Engström, R. Garcia, C. G. B. Peterson, and P. Venge, "Purification and characterization of a human neutrophil lipocalin (HNL) from the secondary granules of human neutrophils," Scandinavian Journal of Clinical and Laboratory Investigation, vol. 54, no. 5, pp. 365-376, 1994.

[10] J. Mishra, M. A. Qing, A. Prada et al., "Identification of neutrophil gelatinase-associated lipocalin as a novel early urinary biomarker for ischemic renal injury," Journal of the American Society of Nephrology, vol. 14, no. 10, pp. 2534-2543, 2003.

[11] K. Mori, H. T. Lee, D. Rapoport et al., "Endocytic delivery of lipocalin-siderophore-iron complex rescues the kidney from ischemia-reperfusion injury," Journal of Clinical Investigation, vol. 115, no. 3, pp. 610-621, 2005.

[12] J. Mishra, C. Dent, R. Tarabishi et al., "Neutrophil gelatinaseassociated lipocalin (NGAL) as a biomarker for acute renal injury after cardiac surgery," The Lancet, vol. 365, no. 9466, pp. 1231-1238, 2005.

[13] A. Clerico, C. Galli, A. Fortunato, and C. Ronco, "Neutrophil gelatinase-associated lipocalin (NGAL) as biomarker of acute kidney injury: a review of the laboratory characteristics and clinical evidences," Clinical Chemistry and Laboratory Medicine, vol. 50, no. 9, pp. 1505-1517, 2012.

[14] D. N. Cruz, S. Gaiao, A. Maisel, C. Ronco, and P. Devarajan, "Neutrophil gelatinase-associated lipocalin as a biomarker of cardiovascular disease: A systematic review," Clinical Chemistry and Laboratory Medicine, vol. 50, no. 9, pp. 1533-1545, 2012.

[15] K. Makris, D. Rizos, N. Kafkas, and A. Haliassos, "Neurophil gelatinase-associated lipocalin as a new biomarker in laboratory medicine," Clinical Chemistry and Laboratory Medicine, vol. 50, no. 9, pp. 1519-1532, 2012.

[16] S. Matsuo, E. Imai, M. Horio et al., "Revised equations for estimated GFR from serum creatinine in Japan," American Journal of Kidney Diseases, vol. 53, no. 6, pp. 982-992, 2009.

[17] J. Kanda, K. Mori, H. Kawabata et al., "An AKI biomarker lipocalin 2 in the blood derives from the kidney in renal injury but from neutrophils in normal and infected conditions," Clinical and Experimental Nephrology, vol. 19, no. 1, pp. 99-106, 2015.

[18] T. Kuwabara, K. Mori, M. Mukoyama et al., "Urinary neutrophil gelatinase-associated lipocalin levels reflect damage to glomeruli, proximal tubules, and distal nephrons," Kidney International, vol. 75, no. 3, pp. 285-294, 2009.

[19] D. Bolignano, V. Donato, G. Coppolino et al., "Neutrophil gelatinase-associated lipocalin (NGAL) as a marker of kidney damage," American Journal of Kidney Diseases, vol. 52, no. 3, pp. 595-605, 2008.

[20] G. P. Otto, J. Hurtado-Oliveros, H. Y. Chung et al., "Plasma neutrophil gelatinase-associated lipocalin is primarily related to inflammation during sepsis: a translational approach," PLoS ONE, vol. 10, no. 4, Article ID e0124429, 2015.

[21] D. Bolignano, G. Coppolino, V. Donato, A. Lacquaniti, C. Bono, and M. Buemi, "Neutrophil gelatinase-associated lipocalin (NGAL): a new piece of the anemia puzzle?" Medical Science Monitor, vol. 16, no. 6, pp. RA131-RA135, 2010.

[22] A. Yndestad, L. Landrø, T. Ueland et al., "Increased systemic and myocardial expression of neutrophil gelatinase-associated lipocalin in clinical and experimental heart failure," European Heart Journal, vol. 30, no. 10, pp. 1229-1236, 2009.

[23] M. Folkesson, M. Kazi, C. Zhu et al., "Presence of NGAL/MMP9 complexes in human abdominal aortic aneurysms," Thrombosis and Haemostasis, vol. 98, no. 2, pp. 427-433, 2007.

[24] A.-L. Hemdahl, A. Gabrielsen, C. Zhu et al., "Expression of neutrophil gelatinase-associated lipocalin in atherosclerosis and myocardial infarction," Arteriosclerosis, Thrombosis, and Vascular Biology, vol. 26, no. 1, pp. 136-142, 2006.

[25] D. Bolignano, G. Basile, P. Parisi, G. Coppolino, G. Nicocia, and M. Buemi, "Increased plasma neutrophil gelatinase-associated lipocalin levels predict mortality in elderly patients with chronic heart failure," Rejuvenation Research, vol. 12, no. 1, pp. 7-14, 2009.

[26] K. Shrestha, A. G. Borowski, R. W. Troughton, J. D. Thomas, A. L. Klein, and W. H. W. Tang, "Renal dysfunction is a stronger determinant of systemic neutrophil gelatinaseassociated lipocalin levels than myocardial dysfunction in systolic heart failure," Journal of Cardiac Failure, vol. 17, no. 6, pp. 472-478, 2011.

[27] S. Lindberg, S. H. Pedersen, R. Mogelvang et al., "Prognostic utility of neutrophil gelatinase-associated lipocalin in predicting mortality and cardiovascular events in patients with STsegment elevation myocardial infarction treated with primary percutaneous coronary intervention," Journal of the American College of Cardiology, vol. 60, no. 4, pp. 339-345, 2012.

[28] P. Falke, A. M. Elneihoum, and K. Ohlsson, "Leukocyte activation: relation to cardiovascular mortality after cerebrovascular ischemia," Cerebrovascular Diseases, vol. 10, no. 2, pp. 97-101, 2000.

[29] L. B. Daniels, E. Barrett-Connor, P. Clopton, G. A. Laughlin, J. H. Ix, and A. S. Maisel, "Plasma neutrophil gelatinaseassociated lipocalin is independently associated with cardiovascular disease and mortality in community-dwelling older adults: the Rancho Bernardo Study," Journal of the American College of Cardiology, vol. 59, no. 12, pp. 1101-1109, 2012.

[30] S. H. Nymo, T. Ueland, E. T. Askevold et al., "The association between neutrophil gelatinase-associated lipocalin and clinical outcome in chronic heart failure: results from CORONA," Journal of Internal Medicine, vol. 271, no. 5, pp. 436-443, 2012.

[31] M. McNairy, N. Gardetto, P. Clopton et al., "Stability of Btype natriuretic peptide levels during exercise in patients with congestive heart failure: implications for outpatient monitoring with B-type natriuretic peptide," American Heart Journal, vol. 143, no. 3, pp. 406-411, 2002.

[32] A. Maisel, "B-type natriuretic peptide levels: diagnostic and prognostic in congestive heart failure: what's next?" Circulation, vol. 105, no. 20, pp. 2328-2331, 2002. 
[33] Y. Takami, T. Horio, Y. Iwashima et al., "Diagnostic and prognostic value of plasma brain natriuretic peptide in nondialysis-dependent CRF," American Journal of Kidney Diseases, vol. 44, no. 3, pp. 420-428, 2004.

[34] M. Hasegawa, J. Ishii, F. Kitagawa et al., "Urinary neutrophil gelatinase-associated lipocalin as a predictor of cardiovascular events in patients with chronic kidney disease," Heart and Vessels, vol. 30, pp. 81-88, 2015. 


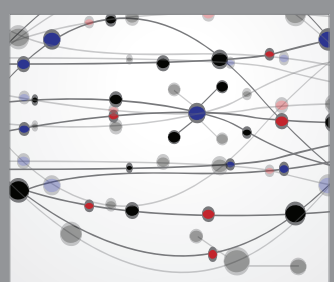

The Scientific World Journal
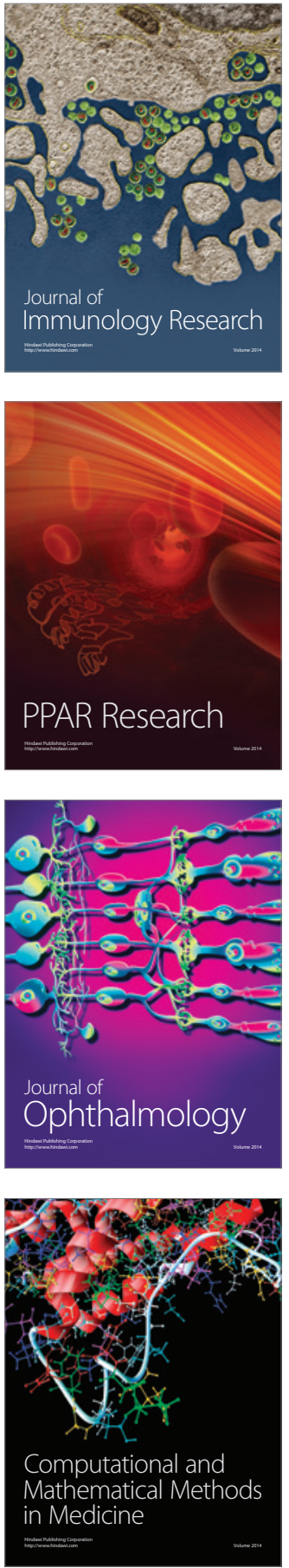

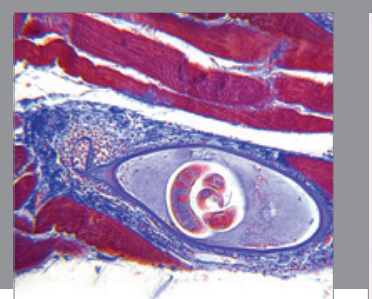

Gastroenterology Research and Practice

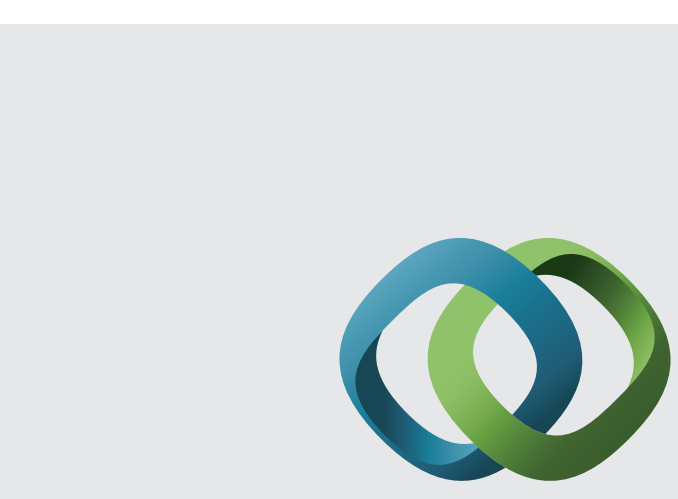

\section{Hindawi}

Submit your manuscripts at

http://www.hindawi.com
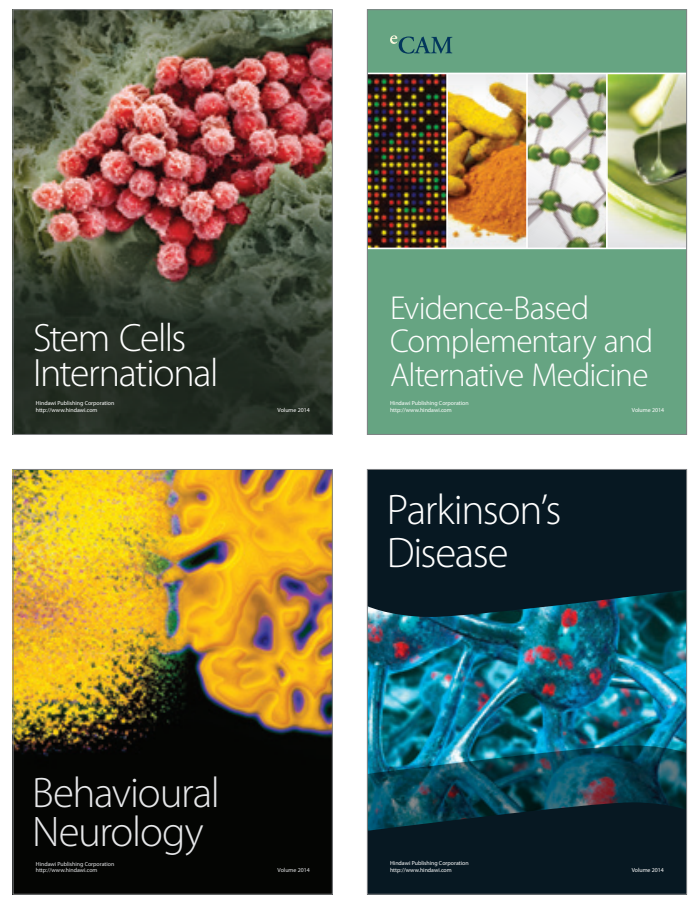
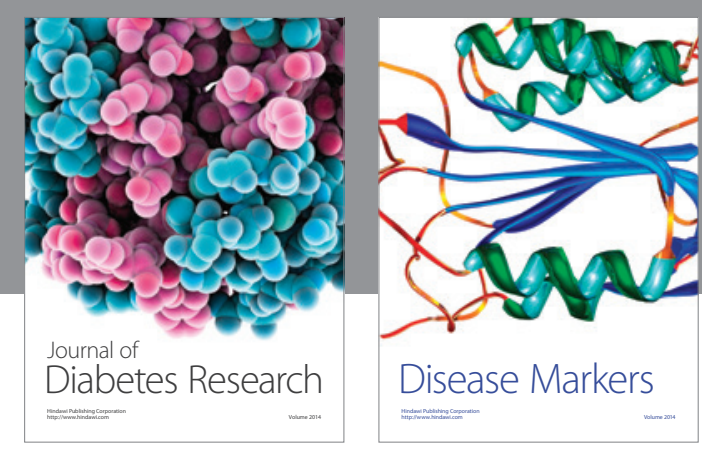

Disease Markers
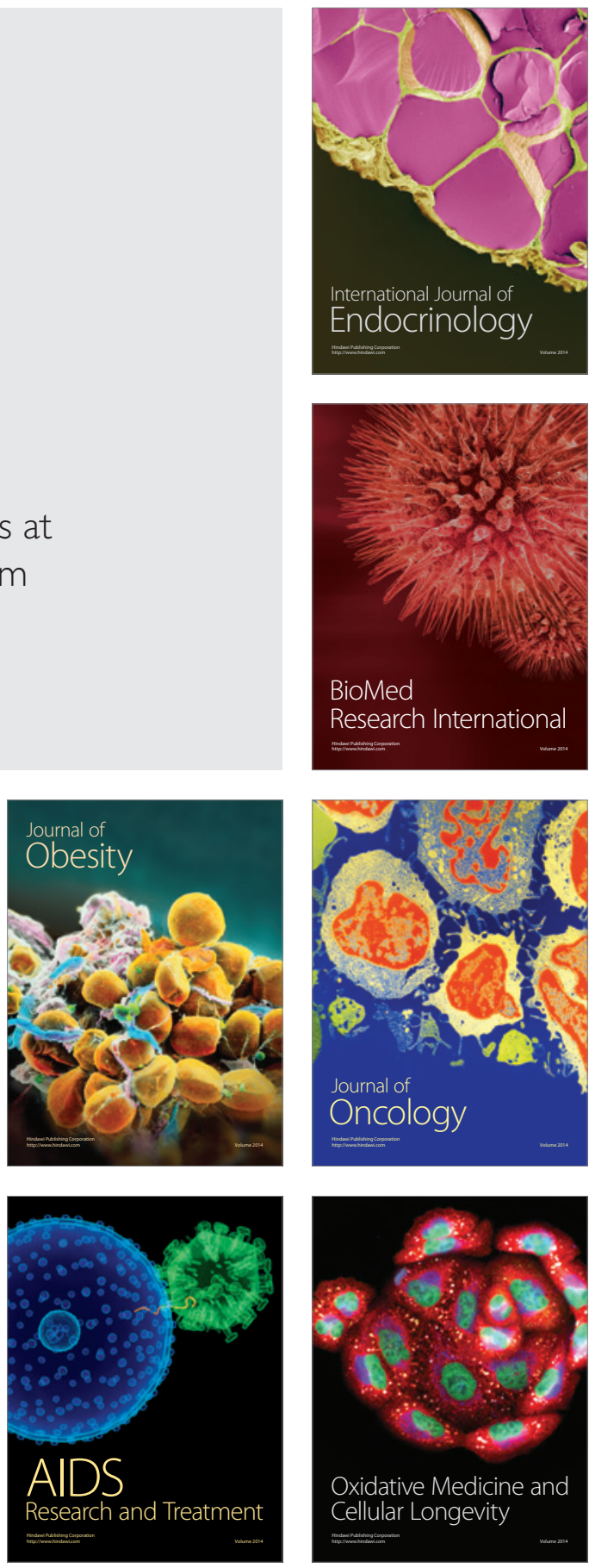